\title{
A Low-cost Distributed Networked Localization and Time Synchronization Framework for Underwater Acoustic Testbeds
}

\author{
Hovannes Kulhandjian \\ Department of Electrical Engineering \\ State University of New York at Buffalo, Buffalo, NY 14260 \\ E-mail: hkk2@buffalo.edu
}

\author{
Tommaso Melodia \\ Department of Electrical and Computer Engineering \\ Northeastern University, Boston, MA 02115 \\ E-mail: melodiadece.neu.edu
}

\begin{abstract}
Localization and time synchronization are both essential services for Internet-connected underwater acoustic testbeds. Although the two are mutually coupled, they are often treated separately. We propose a new low-cost distributed networked localization and time synchronization framework for underwater acoustic sensor networks. The proposal is based on decoupling the two problems and solving first the time synchronization then localization using the same set of messages, i.e., with no additional overhead. A coarse, followed by a finegrained, localization algorithm is adopted to accurately estimate the location of an unknown node. The solution is robust to noisy range measurements. The proposed scheme is implemented in a testbed based on Teledyne Benthos Telesonar SM-975 underwater modems and tested extensively in Lake LaSalle at the University at Buffalo. Experiments and simulations in terms of root mean square error (RMSE) demonstrate that the proposed scheme can achieve a high accuracy for a limited energy budget.
\end{abstract}

\section{INTRODUCTION}

Localization in underwater acoustic (UW-A) networks is essential for geographical routing, medium access control (MAC), autonomous underwater vehicle (AUV) navigation, IP connectivity [1], among other applications. One way to perform localization is to rely on GPS. However, radio waves are highly attenuated underwater. Acoustic communication is the transmission technology of choice for underwater networked systems [2]. The UW-A channel is characterized by slow propagation of acoustic waves, limited bandwidth, high transmit energy consumption, high and variable propagation delays, motion-induced Doppler spread, frequency selective fading and multipath [3]. These characteristics pose severe challenges towards designing robust localization schemes that can achieve the following desirable properties: i) high accuracy, ii) fast convergence, iii) wide coverage, iv) low communication cost, and v) high scalability.

Localization in general requires several sensor nodes with known locations (anchor nodes) with the distance or angle measurements between the anchor nodes and the unknown node. In wireless sensor networks (WSNs) distance or angle can be measured using one of the following methods: i) Angle-of-Arrival (AoA), ii) Received Signal Strength Indicator (RSSI), iii) Time-of-Arrival (ToA), and iv) Time Difference of

Acknowledgment: This work is based upon work supported in part by the US National Science Foundation under grants CNS-1422874 and CNS1126357.
Arrival (TDoA). In underwater acoustic sensor networks (UWASNs) ToA and TDoA are more widely used, as RSSI-based methods suffer from the time varying nature of the UW-A channel and AoA requires an array of transducers, which can be costly to provide in underwater sensor network deployments [4].

A number of localization techniques have been proposed for UW-ASNs [5], [6], [7], which can be categorized into centralized and decentralized; each can be further sub-categorized into estimation-based and prediction-based. In general, centralized localization schemes rely on a central controller, while decentralized schemes can perform localization autonomously.

To determine an accurate estimate of the location of an unknown node it is essential to have the clocks of the sensor nodes synchronized. Nevertheless, when designing underwater range-based localization algorithms often times nodes are assumed to be time synchronized [8], [9], [10], and/or clock skews are not taken into consideration [11].

Very limited research works have addressed joint localization and time synchronization for UW-ASNs. Among them, in [12], JSL, a joint time synchronization and localization protocol with stratification and mobility compensation is presented. However, JSL does not consider range measurement errors and it makes use of a linear least squares quadratic linearization algorithm for localization, which is known to suffer when anchor node location and/or propagation time estimate is not precise [13]. Moreover, to compensate for the stratification effect it assumes the sound speed profile is known a-priori.

In [14], a heuristic algorithm for time synchronization and localization in the UW-A channel is presented. The algorithm relies on the assumption that nodes are equipped with selfnavigation systems and that they provide accurate information, which might not be realistic, as the accuracy of such a system can have a strong impact on the localization algorithm.

It is still desirable to have a robust autonomous networked infrastructure that can provide both localization and time synchronization services at low-cost in practical testbed deployments. To fill this gap, we propose a low-cost distributed networked localization and time synchronization framework for UW-ASNs. The proposal is based on the Internet underwater framework [1], and is implemented in a testbed based on Teledyne Benthos Telesonar SM-975 underwater modems and tested extensively in Lake LaSalle at the University at Buffalo. Experiments in terms of root mean square error (RMSE) demonstrate that the proposed scheme can achieve 
a high accuracy for a limited energy budget, i.e., for a limited number of message exchanges.

The rest of this paper is organized as follows. In Section II, we introduce the problem statement followed by time synchronization and localization algorithms. In Section III, we evaluate the proposed scheme. Finally, in Section IV, we draw the main conclusions.

\section{Problem Statement}

Consider an underwater acoustic sensor network comprised of $N+M$ acoustic modems, $N$ of them deployed at the bottom of a body of water (e.g., ocean, sea or a lake) and the rest on the surface. The nodes that are time-synchronized and localized are called anchor nodes while those that are not are ordinary nodes. In this case, all the $M$ nodes, acting as surface stations, are anchor nodes.

Our objective is to develop a low-cost distributed algorithm that will simultaneously provide time synchronization and localization services for the underwater sensor network in consideration. To achieve that, each ordinary node $n$ will first transmit a small packet $S Y N C_{R E Q}^{n}$ to the anchor nodes requesting time synchronization and localization services. The $S Y N C_{R E Q}^{n}$ packet contains a preamble (an acquisition signal, a linear chirp signal, used for channel probing, symbol synchronization for chip-matched-filtering, Doppler shift estimation and multipath delay spread estimation [4]) and source and destinations $I D$ s along with the sent time stamp of node $n$. This process will be repeated by all the $n \in(1,2, \ldots, N)$ ordinary nodes, each taking turns. After gathering all the $N$ packets from the ordinary nodes each surface station will generate a new packet, $S Y N C_{R E P}^{m}$, containing information on the time the $S Y N C_{R E Q}^{n}$ packet was received from each ordinary node $n$ at surface station anchor node $m$ (denoted by $t_{m, n}^{r}$ ) where $m \in(1,2, \ldots, M)$ denotes the surface station number, the time the new packet is sent by surface station $m$, and its own location coordinates denoted by $\mathbf{p}_{m}=\left[x_{m}, y_{m}, z_{m}\right]^{T}$, where $\mathbf{x}^{T}$ denotes the transpose of vector $\mathbf{x}$.

The time measured by a non-synchronized node $n$ with respect to a reference node $m$ can be expressed as

$$
t_{n}=\alpha_{n} t_{m}+\delta_{n}, \quad n=1,2, \ldots, N, m=1,2, \ldots, M,
$$

where $\alpha_{n} \in \mathbb{R}_{+}$and $\delta_{n} \in \mathbb{R}$ are node $n$ 's clock skew and offset relative to node $m$, respectively. The clock skew and offset are associated with frequency and time difference of the clock, respectively.

Accordingly, the receive time stamp (ToA) at the surface station $m$ from node $n$ can be expressed as

$$
t_{m, n}^{r}=\bar{t}_{n, m}^{s}+\tau_{n, m}+\epsilon_{n, m}, \quad m=1,2, \ldots, M,
$$

where $\bar{t}_{n, m}^{s}=\frac{\left(t_{n, m}^{s}-\delta_{n}\right)}{\alpha_{n}}$ corresponds to the clock synchronized sent time stamp at node $n$, in which $t_{n, m}^{s}$ denotes the unsynchronized sent time stamp registered by node $n$, $\tau_{n, m} \triangleq\left\|\mathbf{p}_{m}-\mathbf{p}_{n}\right\| / c$ is the propagation time from node $n$ to node $m$, in which $\mathbf{p}_{n}=\left[x_{n}, y_{n}, z_{n}\right]^{T}$ is the unknown position coordinates of node $n, c$ is the speed of sound underwater and $\epsilon_{n, m}$ is a measurement error, assumed to be independent and identically distributed following a Gaussian distribution [15].
Similarly, the sent time stamp from the surface station $m$ to node $n$ can be expressed as

$$
t_{m, n}^{s}=\bar{t}_{n, m}^{r}-\tau_{m, n}+\epsilon_{m, n}, \quad m=1,2, \ldots, M,
$$

where $\bar{t}_{n, m}^{r}=\frac{\left(t_{n, m}^{r}-\delta_{n}\right)}{\alpha_{n}}$ is the clock synchronized receive time stamp at node $n$ from node $m$, in which $t_{n, m}^{r}$ denotes the unsynchronized receive time at node $n, \tau_{m, n}$ is the propagation time from node $m$ to node $n$ and $\epsilon_{m, n}$ is a measurement error.

Our objective is to design a low-cost distributed algorithm to compute the clock skews $\left(\alpha_{n}\right)$, clock offsets $\left(\delta_{n}\right)$ and locations $\left(\mathbf{p}_{n}=\left[x_{n}, y_{n}, z_{n}\right]\right)$ of all the $n \in(1,2, \ldots, N)$ ordinary nodes.

We can observe from (2) or (3) that the time synchronization and localization are mutually coupled, i.e., to determine the location of node $n, \mathbf{p}_{n}$, it first needs to be synchronized with an anchor node and vice versa. The proposal is based on decoupling the joint problem by eliminating one of them (i.e., the localization parameter, $\mathbf{p}_{n}$, which is dependent on $\tau_{m, n}$ ).

We first synchronize the clocks of the ordinary nodes with the synchronized ones before performing localization.

\section{A. Time-synchronization}

Assuming the propagation time from node $n$ to node $m$ is the same from node $m$ to node $n$, i.e., $\tau_{n, m}=\tau_{m, n}$, and the skew and offset of node $n$ 's clock are time-invariant during the synchronization process. Adding (2) to (3) we decouple the original problem by eliminating the unknown propagation time variable and thus have

$\left(t_{n, m}^{r}+t_{n, m}^{s}\right)=\alpha_{n}\left(t_{m, n}^{r}+t_{m, n}^{s}\right)+2 \delta_{n}-\alpha_{n}\left(\epsilon_{n, m}+\epsilon_{m, n}\right)$.

Combining all the time stamp information at node $n$ from the $M$ surface stations we can express (4) in matrix form as

$\mathbf{b}=\mathbf{A x}+\boldsymbol{\epsilon}$,
where $\mathbf{b} \triangleq\left[\left(t_{n, 1}^{r}+t_{n, 1}^{s}\right), \ldots,\left(t_{n, M}^{r}+t_{n, M}^{s}\right)\right]_{M \times 1}^{T}$,
$\mathbf{A} \triangleq\left[\left(t_{1, n}^{r}+t_{1, n}^{s}, 2\right)^{T}, \ldots,\left(t_{M, n}^{r}+t_{M, n}^{s}, 2\right)^{T}\right]_{M \times 2}^{T}$,
$\mathbf{x} \triangleq\left[\alpha_{n}, \delta_{n}\right]^{T}, \boldsymbol{\epsilon} \triangleq-\alpha_{n}\left[\left(\epsilon_{n, 1}+\epsilon_{1, n}\right), \ldots,\left(\epsilon_{n, M}+\epsilon_{M, n}\right)\right]^{T}$

Since the measurement error, $\epsilon$, is assumed to follow a Gaussian distribution, we can obtain the maximum-likelihood (ML) estimate of $\mathbf{x}$ by

$$
\hat{\mathbf{x}}_{M L}=\arg \min _{\mathbf{x} \in \mathbb{R}^{2 \times 1}}\|\mathbf{b}-\mathbf{A x}\|_{2}^{2}
$$

For a given set of time stamp information, $\mathbf{A}$ and $\mathbf{b}$, we can solve (6) directly as

$$
\hat{\mathbf{x}}_{M L}=\left(\mathbf{A}^{T} \mathbf{A}\right)^{-1} \mathbf{A}^{T} \mathbf{b} .
$$

Using (2) and $\hat{\alpha}_{n}, \hat{\delta}_{n}$ from (7), the propagation delay between each surface station and node $n$ is estimated as

$$
\hat{\boldsymbol{\tau}}=\mathbf{t}^{r}+\mathbf{B} \hat{\mathbf{y}}
$$

where $\hat{\tau} \triangleq\left[\hat{\tau}_{n, 1}, \hat{\tau}_{n, 2}, \ldots, \hat{\tau}_{n, M}\right]^{T}, \mathbf{t}^{r} \triangleq\left[t_{1, n}^{r}, t_{2, n}^{r}, \ldots, t_{M, n}^{r}\right]^{T}$, $\mathbf{B} \triangleq\left[\left(t_{n, 1}^{s}, 1\right)^{T},\left(t_{n, 2}^{s}, 1\right)^{T}, \ldots,\left(t_{n, M}^{s}, 1\right)^{T}\right]^{T}, \hat{\mathbf{y}} \triangleq \frac{1}{\hat{\alpha}_{n}}\left[\begin{array}{l}-1 \\ \hat{\delta}_{n}\end{array}\right]$.

The rest of the $N-1$ nodes can be synchronized in a similar way. To further improve the synchronization process all the synchronized nodes (i.e., in this case node $n$ ) can also partake in synchronizing the rest of the ordinary nodes. 


\section{B. Localization}

Having synchronized all the deployed nodes, we now address the localization of the individual nodes utilizing the same message exchange information used for time synchronization (i.e., with no additional overhead). We start off with a coarse localization algorithm followed by a fine-grained localization.

1) Coarse Localization (Linear Least Squares): The relative square distance between the ordinary node $n$ and anchor node $m$ is expressed as

$$
\begin{aligned}
d_{n, m}^{2}= & \left\|\mathbf{p}_{n}-\mathbf{p}_{m}\right\|_{2}^{2}=\left(c \cdot \tau_{n, m}\right)^{2}= \\
& =\left(x_{n}-x_{m}\right)^{2}+\left(y_{n}-y_{m}\right)^{2}+\left(z_{n}-z_{m}\right)^{2} .
\end{aligned}
$$

Combining all the relative square distances between node $n$ and $M$ surface stations we express (9) in matrix form as

$$
\operatorname{diag}\left(\mathbf{P}^{T} \mathbf{P}\right)=c^{2} \boldsymbol{\tau} \odot \boldsymbol{\tau},
$$

where $\mathbf{P} \triangleq\left[\mathbf{p}_{n}-\mathbf{p}_{1}, \mathbf{p}_{n}-\mathbf{p}_{2}, \ldots, \mathbf{p}_{n}-\mathbf{p}_{M}\right]_{3 \times M}, \boldsymbol{\tau} \triangleq$ $\left[\tau_{n, 1}, \tau_{n, 2}, \ldots, \tau_{n, M}\right]_{M \times 1}^{T}, \odot$ is element-wise multiplication.

Given the position coordinates of the anchor nodes (i.e., $\mathbf{p}_{1}, \mathbf{p}_{2}, \ldots, \mathbf{p}_{M}$ ) and the estimate of $\boldsymbol{\tau}$ from (8) we would like to find the position coordinates of node $n$, i.e., $\mathbf{p}_{n}$.

We introduce a $j^{\text {th }}$ constraint, an anchor node $j \neq m$, in order to linearize the quadratic form by adding and subtracting $x_{j}, y_{j}$ and $z_{j}$ in (9) as follows [13]

$$
\begin{array}{r}
d_{n, m}^{2}=\left(x_{n}-x_{j}+x_{j}-x_{m}\right)^{2}+\left(y_{n}-y_{j}+y_{j}-y_{m}\right)^{2}+ \\
+\left(z_{n}-z_{j}+z_{j}-z_{m}\right)^{2} .
\end{array}
$$

Expanding and rearranging (11) we get

$$
\begin{aligned}
& \left(x_{n}-x_{j}\right)\left(x_{m}-x_{j}\right)+\left(y_{n}-y_{j}\right)\left(y_{m}-y_{j}\right)+\left(z_{n}-z_{j}\right)\left(z_{m}-z_{j}\right)= \\
= & \frac{1}{2}\left[d_{n, j}^{2}-d_{n, m}^{2}+d_{m, j}^{2}\right]=\frac{1}{2}\left[\hat{d}_{n, j}^{2}-\hat{d}_{n, m}^{2}+d_{m, j}^{2}\right]+\eta_{n, m}, \quad(12)
\end{aligned}
$$

where $d_{m, j}=\sqrt{\left(x_{m}-x_{j}\right)^{2}+\left(y_{m}-y_{j}\right)^{2}+\left(z_{m}-z_{j}\right)^{2}}$ is the distance between node $m$ and node $j, \hat{d}_{n, m}^{2}=\left(c \cdot \hat{\tau}_{n, m}\right)^{2}$, and $\eta_{n, m}$ is range measurement error.

As it does not matter which synchronized node $j$ is used for linearization, we let $j=1$ (i.e., node $j$ is selected as the first surface station and we let $m \in(2,3, \ldots, M))$. We express (12) in matrix form as

$$
\mathbf{q}=\mathbf{W} \mathbf{p}-\boldsymbol{\eta},
$$

where $\mathbf{p} \triangleq\left[\left(x_{n}-x_{1}\right),\left(y_{n}-y_{1}\right),\left(z_{n}-z_{1}\right)\right]_{3 \times 1}^{T}, \mathbf{W} \triangleq$ $\left[\left(x_{2}-x_{1}, y_{2}-y_{1}, z_{2}-z_{1}\right)^{T}, \ldots,\left(x_{M}-x_{1}, y_{M}-y_{1}, z_{M}-z_{1}\right)^{T}\right]^{T}$, $\mathbf{q} \triangleq \frac{1}{2}\left[\left(\hat{d}_{n, 1}^{2}-\hat{d}_{n, 2}^{2}+d_{2,1}^{2}\right), \ldots,\left(\hat{d}_{n, 1}^{2}-\hat{d}_{n, M}^{2}+d_{M, 1}^{2}\right)\right]^{T}$, $\boldsymbol{\eta} \triangleq\left[\eta_{n, 2}, \eta_{n, 3}, \ldots, \eta_{n, M}\right]_{(M-1) \times 1}^{T}$.

If the range measurement error, $\boldsymbol{\eta}$, is assumed to follow a white Gaussian distribution, the ML estimate of $\mathbf{p}$ is

$$
\hat{\mathbf{p}}_{M L}=\arg \min _{\mathbf{p} \in \mathbb{R}^{3 \times 1}}\|\mathbf{W} \mathbf{p}-\mathbf{q}\|_{2}^{2} \text {. }
$$

For a given $\mathbf{W}$ and $\mathbf{q}$, we can solve (14) directly as

$$
\hat{\mathbf{p}}_{M L}=\left(\mathbf{W}^{T} \mathbf{W}\right)^{-1} \mathbf{W}^{T} \mathbf{q} \text {. }
$$

Using (15), node $n$ computes an estimate of its location as

$$
\hat{\mathbf{p}}_{n}=\hat{\mathbf{p}}_{M L}+\mathbf{p}_{1},
$$

where $\mathbf{p}_{1}=\left[x_{1}, y_{1}, z_{1}\right]^{T}$ is the first anchor node's coordinate. The rest of the $N-1$ nodes can be localized in a similar way.

2) Fine-grained Localization (Non-linear Least Squares): The linear least square problem is known to suffer when the anchor node location is not precise [13] and/or the propagation time estimate is not very accurate, which is of the case in underwater localization, as discussed in Sec. III. Therefore, we enhance the localization by solving a variant of the original problem (10) using a non-linear least squares method that can provide a fine-grained location estimation.

Essentially, we would like to minimize the sum of the squared errors of the distances, which is expressed as

$F\left(x_{n}, y_{n}, z_{n}\right)=\sum_{m=1}^{M} f_{m}\left(x_{n}, y_{n}, z_{n}\right)^{2}=\sum_{m=1}^{M}\left(d_{n, m}-\hat{d}_{n, m}\right)^{2}$.

Minimizing the sum of the square error is a common problem in applied mathematics and a number of algorithms are available to solve it efficiently [16]. Among them the Newton method is one of the widely used ones. However, the Newton algorithm requires a 'good' initial guess for $\hat{\mathbf{p}}_{n}$, which is obtained from the least square method (16). The fine-grained localization algorithm is given in direct implementation form in Algorithm 1, where $\mathbf{f} \triangleq$ $\left[f_{1}\left(x_{n}, y_{n}, z_{n}\right), \ldots, f_{M}\left(x_{n}, y_{n}, z_{n}\right)\right]_{M \times 1}^{T}$ and $\mathbf{J}$ is the Jacobian matrix [16]. A stopping criteria, $\varepsilon$, is selected depending on the desired level of accuracy.

\begin{tabular}{l}
\hline Algorithm 1: Fine-grained Localization Algorithm \\
\hline \hline 1) $k:=0$; initialize $\hat{\mathbf{p}}_{n}^{1}=\left[\hat{x}_{n}, \hat{y}_{n}, \hat{z}_{n}\right]^{T}$ from (16). \\
2) $k:=k+1$. \\
2a) Compute $\hat{\mathbf{p}}_{n}^{k+1}=\hat{\mathbf{p}}_{n}^{k}-\left(\mathbf{J}_{k}^{T} \mathbf{J}_{k}\right)^{-1} \mathbf{J}_{k}^{T} \mathbf{f}^{k}$. \\
3) Repeat Step 2 until $\left\|\hat{\mathbf{p}}_{n}^{k+1}-\hat{\mathbf{p}}_{n}^{k}\right\|_{2} \leq \varepsilon$. \\
\hline
\end{tabular}

In the existing range-based localization protocols [12], [17], [18], at least $N \times M$ message exchanges are necessary to synchronize $N$ nodes, while the proposed scheme only requires at least $N+M$. Therefore, it can provide considerable energy savings in a large-scale distributed network.

\section{A. Simulation Results}

\section{PERFORMANCE EVAluATION}

Simulations were preformed to study the performance of proposed time synchronization and localization algorithms discussed in Sec. II. Four anchor nodes were statically placed in the center of a region with a surface area $400 \mathrm{~m} \times 400 \mathrm{~m}$ separated by $200 \mathrm{~m}$ from each other. The ordinary node was placed at random locations with depth ranging from $5 \mathrm{~m}$ to $100 \mathrm{~m}$. The following parameters were randomly selected using a uniform distribution during the simulations; measurement error variance $\sigma^{2}$ from $0 \mathrm{~dB}$ to $-50 \mathrm{~dB}$, the clock skew $\alpha$ from $-50 \mathrm{ppm}$ to $50 \mathrm{ppm}$, clock offset $\delta$ from $-5 \mathrm{~s}$ to $5 \mathrm{~s}$, while the average speed of sound $c=1500 \mathrm{~m} / \mathrm{s}$ was considered constant. In case the sound speed profile is known a-priori we can use the empirical sound speed formula [3] to compensate the "stratification effect", as discussed in [12]. The number of anchor nodes $M=4$, and stopping criteria, $\varepsilon=0.1 \mathrm{~m}$. All the simulation results were averaged over 10,000 independent 


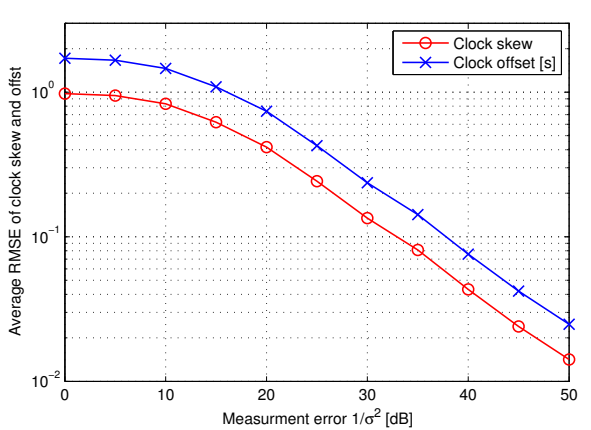

Fig. 1: Average RMSE of clock skew and offset versus measurement error.

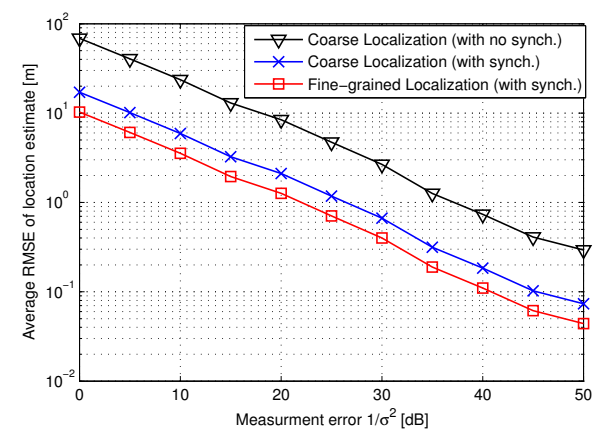

Fig. 2: Average RMSE of location versus measurement error.

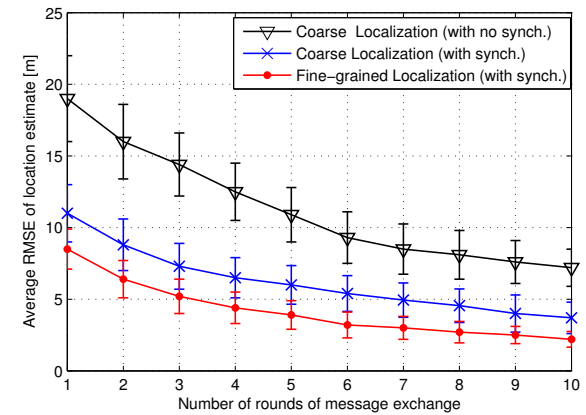

Fig. 3: Average RMSE of location versus number of rounds of message exchange. runs, i.e., 100 random locations of ordinary node were selected and for each location it was repeated 100 times.

In Fig. 1 we plot the average RMSE of the clock skew of ordinary node and offset versus measurement error. As the measurement error decreases the algorithm estimates the clock skew and offset with high accuracy.

In Fig. 2 we plot the average RMSE of the location of ordinary node versus location measurement error for a) coarse localization with no synchronization compensation (i.e., assuming $\alpha_{n}=1, \delta_{n}=0$ ), b) coarse localization with synchronization, and c) fine-grained localization with synchronization. We observe that without synchronization localization performs very poorly. The fine-grained localization shows substantial improvement over the coarse localization scheme with synchronization. As the measurement error decreases the performance improves considerably.

\section{B. Experimental Evaluation}

Experiments were conducted in Lake LaSalle using four Telesonar SM-975 modems. The actual deployment of the four UW-A modems is shown in Fig. 4, each Telesonar SM-975 modem was attached to a buoy along with an anchor. Three anchor nodes labeled Node 1,2,3 were deployed $1 \mathrm{~m}$ below the surface, while the ordinary node (Node 4) was deployed $3 \mathrm{~m}$ below the surface at the exact locations shown in Fig. 4. The average depth of the lake was about $4.5 \mathrm{~m}$. In practice, only three modems are sufficient for underwater localization, as the depth parameter can accurately be measured using either a depth sensor [5], [12], or ranging methods (e.g., using round-trip time (RTT)). The latter method was applied in our case. The actual location of the nodes (longitude and latitude, which can be converted to Cartesian coordinates [19]) were determined using a GPS receiver with an accuracy of $\pm 1.8 \mathrm{~m}$.

One of the unique features of the Telesonar SM-975 modems is the serial binary control protocol called Modem Management Protocol (MMP) [20] that can provide the user with the packet send and receive time stamp information, which was utilized during the experiments.

A bit rate of $800 \mathrm{bits} / \mathrm{s}$ with multiple frequency shift keying (MFSK) modulation scheme was used to conduct the experiments with a transmit power level set at $4.8 \mathrm{~W}$. A laptop, on an inflatable boat, was used to coordinate the transmissions of the $S Y N C_{R E Q}$ and $S Y N C_{R E P}$ packets through a serial port interface. From one to ten rounds of information were

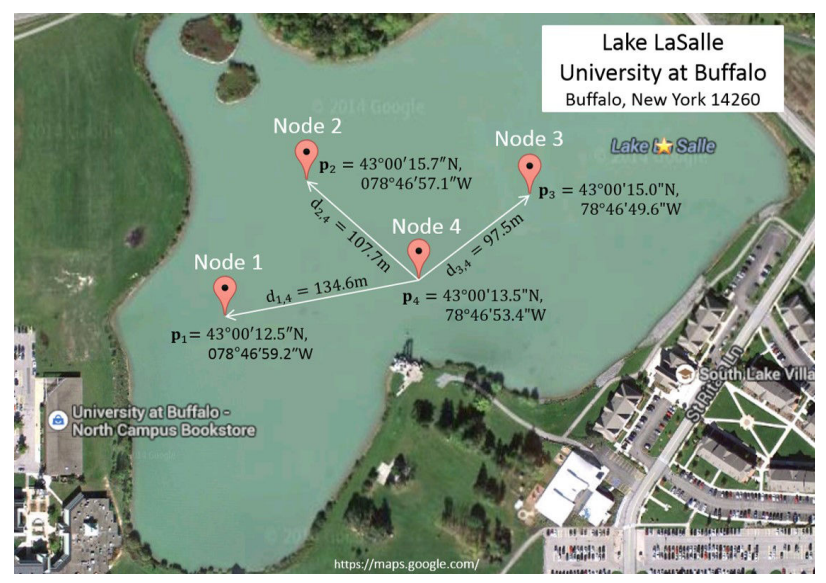

Fig. 4: Testbed deployment in Lake LaSalle at the University at Buffalo.

exchanged and each round was repeated 10 times. Each round consists of four packets exchange (i.e., $S Y N C_{R E Q}+3$ $\left.S Y N C_{R E P}\right)$. The average values are presented in the plot.

In Fig. 3 we plot the average RMSE of the location of ordinary node versus the number of rounds of message exchange for a) coarse localization with no time synchronization compensation, b) coarse localization with synchronization, and c) fine-grained localization with synchronization. From the observed high estimation error it is evident that accurate localization necessitates clock synchronization. In addition, we can see that the fine-grained localization can provide considerable improvement over the coarse localization with synchronization. Obviously, increasing the number of rounds of message exchanges improves the accuracy. Apart from GPS accuracy, other major factors that play a key role on the accuracy of our estimates are the relatively short distance of the nodes and the high multipath in the shallow water channel [4]. The multipath can have a strong impact on the precision of time stamp estimation.

\section{CONCLUSiOnS}

We presented a new low-cost distributed networked localization and time synchronization scheme, which we implemented in a testbed based on Telesonar SM-975 underwater modems and tested extensively in Lake LaSalle. Experiments and simulations in terms of RMSE demonstrate that the proposed scheme can achieve a high accuracy for a limited energy budget. 


\section{REFERENCES}

[1] Y. Sun and T. Melodia, "The Internet Underwater: An IP-compatible Protocol Stack for Commercial Undersea Modems," in Proc. of ACM Intl. Conf. on UnderWater Networks and Systems (WUWNet), Kaohsiung, Taiwan, November 2013.

[2] H. Kulhandjian, T. Melodia, and D. Koutsonikolas, "CDMA-based Ana$\log$ Network Coding through Interference Cancellation for Underwater Acoustic Sensor Networks," in Proc. of ACM Intl. Conf. on UnderWater Networks and Systems (WUWNet), Los Angeles, CA, USA, Nov. 2012.

[3] T. Melodia, H. Kulhandjian, L. Kuo, and E. Demirors, "Advances in underwater acoustic networking," in Mobile Ad Hoc Networking: Cutting Edge Directions, S. Basagni, M. Conti, S. Giordano, and I. Stojmenovic, Eds. Inc., Hoboken, NJ: John Wiley and Sons, 2013, pp. 804-852.

[4] H. Kulhandjian, T. Melodia, and D. Koutsonikolas, "Securing Underwater Acoustic Communications through Analog Network Coding," in Proc. of IEEE Intl. Conf. on Sensing, Communication, and Networking (SECON), Singapore, June 2014.

[5] M. Erol-Kantarci, H. T. Mouftah, and S. Oktug, "A Survey of Architectures and Localization Techniques for Underwater Acoustic Sensor Networks," IEEE Communications Surveys \& Tutorials, vol. 13, no. 3, pp. 487-502, Third Quarter 2011.

[6] H. Tan, R. Diamant, W. Seah, and M. Waldmeyer, "A survey of techniques and challenges in underwater localization," Elsevier Journal of Ocean Engineering, vol. 38, no. 14-15, pp. 1663-1676, October 2011.

[7] V. Chandrasekhar, W. K. Seah, Y. S. Choo, and H. V. Ee, "Localization in underwater sensor networks - survey and challenges," in Proc. of ACM International Conference on Mobile Computing and Networking (MobiCom), New York, NY, USA, Sep. 2006, pp. 33-40.

[8] A. Y. Teymorian, W. Cheng, L. Ma, X. Cheng, X. Lu, and Z. Lu, "3D Underwater Sensor Network Localization," IEEE Transactions on Mobile Computing, vol. 8, no. 12, pp. 1610-1621, April 2009.

[9] D. Mirza and C. Schurgers, "Motion-aware self-localization for underwater networks," in Proc. of ACM Intl. Workshop on Underwater Networks, San Francisco, California, USA, Sep. 2008, pp. 51-58.

[10] A.-K. Othman, "GPS-less localization protocol for underwater acoustic networks," in Proc. IEEE Wireless and Optical Communications Networks, Asia Pacific, Singapore, May, 2008.

[11] J. Zheng, K. Lui, and H. So, "Accurate three-step algorithm for joint source position and propagation speed estimation," IEEE Journal of Signal Processing, vol. 87, no. 12, pp. 3096-3100, October 2007.

[12] J. Liu, Z. Wang, M. Zuba, Z. Peng, J. Cui, and S. Zhou, "JSL: Joint time synchronization and localization design with stratification compensation in mobile underwater sensor networks," in Proc. of IEEE Conf. on Sensor, Mesh and Ad Hoc Communications and Networks (SECON), Seoul, Korea, June 2012.

[13] F. Izquierdo, M. Ciurana, F. Barcelo, J. Paradells, and E. Zola, "Performance evaluation of a TOA-based trilateration method to locate terminals in WLAN," in Proc. of IEEE Intl. Symposium on Wireless Pervasive Computing, January 2006, pp. 1-6.

[14] R. Diamant and L. Lampe, "Underwater Localization with TimeSynchronization and Propagation Speed Uncertainties," IEEE Transactions on Mobile Computing, vol. 12, no. 7, pp. 1257-1269, July 2013.

[15] D. L. Codiga, J. A. Rice, and P. A. Baxley, "Joint distributed synchronization and positioning in UWB ad hoc networks using ToA," IEEE Trans. Microw. Theory Tech., vol. 54, no. 4, pp. 1896-1911, June 2006.

[16] S. C. Chapra and R. P. Canale, Numerical methods for engineers. New York, NY: McGraw Hill, Higher Education, 2010.

[17] X. Cheng, H. Shu, Q. Liang, and D. H.-C. Du, "Silent Positioning in Underwater Acoustic Sensor Networks," IEEE Transactions on Vehicular Technology, vol. 57, no. 3, pp. 1756-1766, May 2008

[18] V. Chandrasekhar and W. Seah, "An area localization scheme for underwater sensor networks," in Proc. of IEEE/MTS OCEANS, Singapore, May 2007, pp. 1-8.

[19] A. El-Rabbany, Introduction to GPS: the global positioning system. Boston, MA, USA: Artech House, second edition, 2006.

[20] H. Kulhandjian, L. Kuo, T. Melodia, D. A. Pados, and D. Green, "Towards Experimental Evaluation of Software-Defined Underwater Networked Systems," in Proc. of IEEE Underwater Communications Conf. and Workshop (UComms), Sestri Levante, Italy, September 2012. 